Peters für die Astronomischen Nachrichten zugesandt hatte, machte dieser mich darauf aufmerksam, dass Herr Professor Grunert im Januarhefte des Jahrganges 1856 der Sitzungsberichte der mathematisch-naturwissenschaltlichen Classe der Akademie der Wissenschaften in Wien eine ähnliche Auflösung gegeben habe. Nach demnächst vorgeoommener An. sicht der letztern finde ich auch Ähnlichkeiten auf der einen, Unterschiede auf der andern Seite. Ich habe auch das dort gegebene Beispiel nach meiner Auflüsung berechnet, und fincle $\boldsymbol{E}_{1}=28^{\circ} 39^{\prime} 46^{\prime \prime} 68 \quad \boldsymbol{E}_{\mathrm{2}}=43^{\prime \prime} 33$, beide mit Girunert's ersten und letzten Resultate vollkonmen übereinstimmend.

Berlin 1862 Febr. 21 .

Wolfers.

\title{
Osservazioni dell' Ecclisse parziale del Sole del 31 Decembre 1861,
} fatte nell Osservatorio del Coll. Romano col calcolo relativo fatto dal P. Rosa, assistente allo stesso Osservatorio.

(Hierzu die beifolgende Steindrucktafel Fig. 1.)

$$
\text { Osservatore P. Secchi. }
$$

Equatoreale di Merz Lurghezza focale 14 piedi. Apertura 9 pulcici. Ingrandimento 200. Cronometro di Dent.

Principio

T. M. di Roma

Macchia (a)

$2^{\mathrm{b}} 53^{\mathrm{m}} 22^{\mathrm{s}} 79 \%$

$258 \quad 5,32$

Macchia $(A)\left\{\begin{array}{l}\text { Penombra I. L. } \\ \text { Macchia I. L. } \\ \text { Centro } \\ \text { Macchia II. L. } \\ \text { Penombra II. L }\end{array}\right.$

Macchia $d, e$

$\begin{array}{llll}2 & 59 & 33,78\end{array}$

$2 \quad 59 \quad 59,21$

Macchia $3^{3} \cdot 4^{3}$ 1. contatto

11. contatio

Macchia $(B)\left\{\begin{array}{l}\text { 1. contatto } \\ \text { Centro } \\ \text { 11. contatto }\end{array}\right.$

$334 \quad 47,49$

33 I 12,42

Macchia (C) $\left\{\begin{array}{llll}\text { I. contatto } & 3 & 35 & 31,87 \\ \text { Centro } & 3 & 35 & 55,80\end{array}\right.$

lil. contatto 33615,25

Macchia (c) $\left\{\begin{array}{l}\text { l. contatto } \\ \text { Centro } \\ \text { contatto }\end{array}\right.$
Osservatore P. Rosa.

Equatoriale di Cauchoix. Lurghezza focale 7 piedi. Apertura 6 pulcici. Ingrandimento 200. Pendulo Dent.

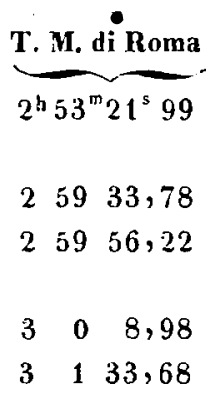

$\begin{array}{lll}333 & 1,57\end{array}$

33323,41

$334 \quad 47,92$

33521,33

33554,71

$\begin{array}{lll}3 & 39 & 24,73\end{array}$

$\begin{array}{lll}3 & 39 & 36,70\end{array}$

II P. Rosa ha fatto i sequenti calcoli nelle osservazioni precendenti. Dalle Tavole del Sig. Leverrier si ottiene

$$
\frac{\text { T. M. di Parigi }}{1861 \text { Dec. } \begin{array}{r}
30,5 \\
31,0 \\
31,5
\end{array}}
$$

1861 Dec. 30,5

31,0

31,5

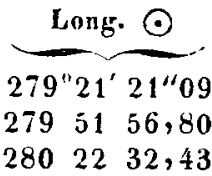

$\frac{\log r}{9,9926754}$
9,9926747
9,9926743

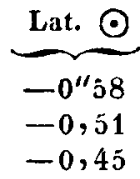

AR. $\odot$

$280^{\circ} 10^{\prime} 54^{\prime \prime} 42$

$280 \quad 44 \quad 5,06$

$\begin{array}{lll}281 & 17 & 14,49\end{array}$

Semidiam $\odot$

$16^{\prime} 16^{\prime \prime} 33$

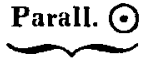

$9 / 10$
Dist. P. N. $\odot$

$113^{\circ} 7^{\prime} 38^{\prime \prime} 19$

113525,51

11335,85

Obliquitá app. Ecclit.

$23^{\circ} 27^{\prime} 26^{\prime \prime} 74$

') Si dove toglierc circa $1^{\text {s }}$ perché il lembo del sole exa ondulato, e non si potè precisare bene l'entrata.

A.S. 


$$
\begin{array}{lllll}
\text { AR. } \odot=280^{\circ} 44^{\prime} & 5^{\prime \prime} 06 & +[3,2988606] t_{1} & -[9,81624] t_{1}{ }^{2} \\
\text { D.p. } \odot=113 & 5 & 25,51 & -[2,1340814] t_{1} & -[0,54282] t_{1}{ }^{2}
\end{array}
$$

Dove rappresentando $\tau$ il tempo medio di Greenwich in frazione di giorno

$$
t_{1}=2 \tau+0,012977 \mathrm{~s} \text {. }
$$

Dalle Tavole del Sig. Hansen pel 31 Decembre $2^{h} 53^{\mathrm{m}} 21^{\mathrm{s}} 99$ T. M. Rom.

$$
\underbrace{\text { Long. L. } \mathbb{C}}_{280^{\circ} 2^{\prime} 37^{\prime \prime} 72} \underbrace{\text { Mov. in } 0^{\circ} 01}_{+8^{\prime} 42^{\prime \prime 72}} \underbrace{\text { Lat. } \lambda \mathbb{C}}_{+0^{\circ} 31^{\prime} 38^{\prime \prime} 02} \underbrace{\text { Mov. in } 0^{8} 01}_{+48^{\prime \prime} 16} \quad \underbrace{\text { Semid. } \mathbb{S}}_{16^{\prime} 21^{\prime \prime} 49} \underbrace{\text { Parall. } \mathbb{C}}_{59^{\prime} 55^{\prime \prime 97}}
$$

$$
\text { AR. } \mathbb{C}=280^{\circ} 53^{\prime} 4^{\prime \prime} 70 \quad \text { D. } \mathbb{C}=112^{\circ} 33^{\prime} 3^{\prime \prime} 73
$$

Obliquita app. dell' Ecclittica $23^{\circ} 27^{\prime} 26^{\prime \prime} 07$.

$\delta \mathrm{AR} .=[0,033288] \delta \boldsymbol{L}-[8,910593] \delta \lambda$

$\delta \mathrm{D} .=-[8,8760297] \delta \boldsymbol{L}-[9,998761] \delta \lambda$

Movimento della AR. $\mathbb{C}$ in $0^{5} 01=+560^{\prime \prime} 340$

Movimento della Dist.Palar. in 0,01 $=-87,315$
Supponendo esatti questi dati a $2^{\mathrm{h}} 53^{\mathrm{m}} 21^{\text {s}} 99$ T. M. Roma il lembo della Luna era roprapposto al lembo Solare di $1^{4} 75$ il che é necessario fisiamente.

Si é dedutta poi la sequente equazione di condizione

$$
\begin{array}{rllll}
1^{\prime \prime} 75+\delta s= & -0,42943 \delta t & -0,52257 \delta \tau & -0,78215 \delta \alpha & +0,53955 \delta \beta \\
& +0,77599 \delta \alpha^{\prime} & -0,537099 \delta \beta^{\prime} & +0,003333 \nu & +0,87404 \delta p
\end{array}
$$

Dove

(1) $\left\{\begin{array}{l}\delta t \text { é la correzione del tempo medio da noi osservato. } \\ \delta r==\text { della nostra longitudine da Greenw. }\left(-49^{n 1} 56^{\mathrm{s}} 13\right) \text {. }\end{array}\right.$

(2) $\left\{\begin{array}{lll}\delta s== & =\text { della somma dei simidiametri. } \\ \delta \alpha== & =\text { dell AR. della Luna. } \\ \delta \beta== & =\text { della Dist. Polare della Luna. } \\ \delta \alpha^{\prime}== & \text { dell' AR. del Sole. } \\ \delta \beta^{\prime}== & \text { della Dist. Polare del Sole. } \\ \delta p== & =\text { della differenza della parallassi oriz. equator. }\end{array}\right.$

ע Numero delle unità di aumento nel denominatore delle schianianento terrestre che vi suppone 3 . $^{\circ}$.

Le quantita (1) sono espreste is secondi di tempo medio.

$=\quad$ = (2) sono variazioni nei dati geocentrici ed espreste in secondi di arco.

Le si veglia adottare la parallasi del Sole del Sig. Encke 8"72 ed il semidiametro solare risultante dalle osservazioni di Greenwich $16^{\prime} 18^{\prime \prime} 20$ si troverebbe dalla supperiore equazione di condizione supponendo il resto dalle variazioni nulle

Roma 1862 Febbraio 26.

$$
\delta t=-7^{5} 45
$$

il che non é punto probabile poichè la nostra osservazione é certamente esatta dentro il secondo che é quanto dire é esatta quanto lo passano essere somiglianti osservazioni.

\section{A. Seceli.}

\section{Schreiben des Herrn Bulard, Directors der Sternwarte in Algier, an den Herausgeber.}

J'ai l'honneur de vous envoyer le résultat de mes observations sur l'eclipse $\odot$ du 31 Décembre.

J'étais parti d'Alger dans l'espoir de pouvoir me rendre sur la route de Ghadmes. Latitude $30^{\circ} 1$. Longit. de Greenwich $7^{\circ} 14$.

J'ai été obligé de m'arrêter a Ouargla à une Latitude Nord $1^{\circ} 56^{\prime} 9^{\prime \prime}$ de celle que je voulais atteindre. La route de Ghadmes n' est pas sur en ce moment il $y$ en des agitateurs dernierement et encore aujourd' hui. Je loge dans une maison en terre un gombi qui appartient a Sid La Lee, Caïd de Ouargla et qui a été incendié par Mohammed ben Abdalah qui lui mème a été pris tout recenment et condamné à mort. Personne n'aurait pu laire plus. Je suis parti contre la volonté de tout le monde on $m$ ' a mis de grandes difficultées pour m'empécher d'arriver parceque la route n'est pas sure voila 29 jours que nous voyageons et 15 a chameaux 